\title{
Supporting Cooking Tasks by a Smart Formulation Table
}

\author{
Wolfgang Maass ${ }^{1,2}$ \\ ${ }^{1}$ Research Center for Intelligent Media \\ Furtwangen University, Germany \\ ${ }^{2}$ Institute of Technology Management, \\ University of St. Gallen, Switzerland
}

\author{
Andreas Filler \\ Research Center for Intelligent Media \\ Furtwangen University, Germany
}

\begin{abstract}
Kitchen environments are target of many Ubiquitous Computing prototypes that investigate the application of technologies in real-world situations. In this paper, we present a design-driven approach that carefully analyzes domain theories, identifies requirements for human task support, derives services accordingly, and uses these descriptions for guiding implementations. We focus on how preparation and concocting tasks can be supported by a formulation table with integrated scaling mechanism and a set of dedicated information services.
\end{abstract}

tangible user interfaces, intelligent environment, design method

\section{INTRODUCTION}

A kitchen is an environment equipped with different tools and services that assist individuals and groups in different situations, such as cooking, washing the dishes, breakfast preparation, and also communications. Electronic tools and services started as simple aids, e.g., can opener, mixer, or refrigerators, but increasingly become more complex assistive devices [1-3].

Cooking situations are typically characterized by some planned behavior that follows scripts described by recipes. These recipes are either explicit or implicit. Implicit recipes are just remembered by individuals while explicit recipes are externally documented in books, on notes, or by digital media. Cooking can be a social event but it is an individual task most of the time.

Otl Aicher distinguishes four task categories with about 250 tasks that are performed in a kitchen: (1) preparation, (2) concocting, (3) cooking, and (4) arranging [4]. Here we focus on two tasks: weighing (preparation task) and mixing (concocting task). Additionally we also look at the recipe selection and recipe tracking task. Following Aicher, cooking is best supported if the main activity area requires little movements and provides enough space in immediate reach. He distinguishes three main activity areas: (1) formulation area for preparation and concocting, (2) cooking area, and (3) washing area. Formulation areas are provided by tables, cooking areas by stoves, and washing areas by tables, sinks, and/or dish washers.
In this article, we focus on the formulation area and how it can be enhanced by non-intrusive information services that support weighing and mixing tasks. On technological level, we investigate whether Ubiquitous Computing technologies, Semantic Technologies, and Internet of Things (IoT) technologies can be used to positively support preparation and concocting situations in a way that does not interfere with traditional cooking behaviors.

We present how requirements are translated into system architectures and a prototype. Empirical studies are current work.

\section{REQUIREMENTS}

In our project, we have selected several functional and non-functional user requirements related to preparation and concocting situations. These requirements represent a selection for complete cooking processes encompassing all four situations.

Recipes are plans for cooking a meal that describe in which way and order ingredients have to be processed. Web sites, such as allrecipes.com, foodnetwork.com, and chefkoch.de, offer huge amounts of recipes. Our system shall be able to automatically retrieve recipes from online repositories so that it can be used for matching with available ingredients and active support of preparation, concocting, cooking and arranging situations. This means passive recipe descriptions from online repositories shall be automatically transformed into executable plans that are used in complete cooking situations.

In the most generic situation, a cook matches available ingredients with recipes, determines missing ingredients or selects alternative recipes. This situation has two extreme cases. Either no ingredients are available for a particular recipe or all ingredients are available. This decision making process for selecting an appropriate recipe shall be supported by an information service that indicates which ingredients are missing.

In a concocting situation, a cook often weighs and mixes ingredients. Today (s)he uses stand-alone scales that require additional work steps. Hence, we required the system to 
integrate a weighing service directly into the concocting situation.

A more basic requirement is that the system shall recognize and track the location of ingredients and give advice if something interferes with the recipe. This means that all representations are directly grounded in physical ingredients. Furthermore the intelligent environment shall support a cook by non-intrusive visual markers that give references to ingredients. The whole system shall be easy to learn and map onto general design principles.

Weighing and mixing tasks shall be supported by a set of information services that are presented in close proximity to referred entities. Special emphasis has been laid on the cooking task of mixing ingredients, e.g., flour, milk, and sugar.

Adding functionality to environments is a tricky task. Each new function requires change of behaviors. Hence, our formulation assistance system shall actively assist a cook with as little additional tasks as possible. In the best case, the system shall help to reduce secondary tasks, such as search, using, cleaning, and storing a scale. This also requires full integration of the system into existing environments and no additional devices to be handled by a cook, such as remote controls or external displays.

Because formulation areas are defined by tables, we have collected requirements according to Fishkin's twodimensional taxonomy for Tangible User Interfaces [5]. On the embodiment dimension, our formulation environment shall be "nearby", i.e., the output of information services shall take place near the input object. Input objects are ingredients and tools, such as bowls. On the metaphor dimension the system shall be classified as "noun and verb", i.e., if, for instance, an ingredient is taken by the cook to fill a bowl, the system shall understand the activity, the ingredient itself, and the resulting state of the activity

Requirements for system design are two-folded. First, the system architecture shall be lean based on smart combination of simple input data. Second, the system shall be robust and cost-effective.

\section{MODEL}

According to these requirements, we started with a table as the basic environment for preparation and concocting situations. With focus on weighing and mixing tasks led, derived several invariants always valid in this intelligent formulation environment:

1. An ingredient that is poured into a bowl is lifted from the table

2. Only one ingredient is poured at a time

3. All ingredients used for cooking are placed on the table

Based on these requirements and invariants, we ran through several recipes and evaluated possibilities for information service support related to recipe selection, recipe tracking, weighing, and mixing tasks. Tasks were broken down into activities that are performed by cooks in interaction with recipes, ingredients, and various cooking tools.

For the recipe selection tracking task, we identified the following activity: looking up the recipe from online repositories. The recipe tracking task consists of two activities: (1) identification of the next task in the recipe and (2) determination of a particular ingredient. Both activities can be supported if the next step in the recipe is automatically highlighted and if appropriate ingredients are visually marked.

In traditional settings, ingredients are weighed according to recipe requirements on an additional scale that is placed on the table. The goal of a weighing task is that a precise amount of an ingredient is extracted and poured into an additional container. Hence, a weighing task can be dissected into several activities: (1) preparation of a scaling device, (2) preparation of a container, (3) grasping an ingredient, (4) pouring this ingredient into the container on top of the scale till a specific amount is reached, (5) putting back the ingredient, (6) retrieving the container from the scale, and (7) removing the scale. We found that activities 1,6 , and 7 can be eliminated if the scale is integrated into the table.

In the same way, we analyzed the mixing task that consists of the following basic activities: (1) adding a defined amount of an ingredient to a bowl and (2) manually mixing ingredients in a bowl. A mixing task uses results of weighing tasks but sometimes ingredients can be directly used in mixing tasks without weighing, e.g., pinch of salt and one liter of milk. This analysis shows that activity 1 is closely related to a weighing task and activity 2 is mainly done manually by the cook. Activity 2 might get support by information services, e.g., based on measurements of time, temperature, and consistency measurements, which is beyond the scope of this article.

Based on this careful domain analysis we designed supporting information services for weighing and mixing tasks. For the recipe selection task:

- Recipe retrieval service: retrieves recipes by considering ingredients already placed on the preparation table. The cook is informed for each recipe which ingredients are missing on the formulation table (supports activity 1 of the recipe selection task).

Recipe tracking task:

- $\quad$ Recipe tracking service: helps a cook to keep track of the next step (activity 1 recipe tracking task)

- Ingredient identification service: helps the user to identify an ingredient by markers (activity 2 recipe tracking task).

For the weighing task, we defined the following information services

- Weighing service: integrated weighing service with weight marker 
- Alarm service: indicate if an inappropriate ingredient is taken.

The mixing task reuses services from other tasks, such as the recipe tracking service, ingredient identification service, weighing service, and alarm service.

\section{System Design OF THE SMART COOKING ENVIRONMENT ARCHITECTURE}

A basic system-level requirement for the Smart Cooking Environment Architecture (SCEA) is that it shall be open for future services for all four phases, i.e., preparation, concocting, cooking, and arranging. Furthermore SCEA is required to support easy access to online data repositories. All data processed within SCEA shall be semantically annotated so that higher-order services, such as logical inferencing and natural language processing can be supported in the future.

The physical environment was constrained by our basic requirement to provide a non-intrusive environment. Formulation tasks are performed on a table. Therefore a table environment fits naturally. Removing activities 1,6 , and 7 of the weighing task requires that the scale is integrated into the table.

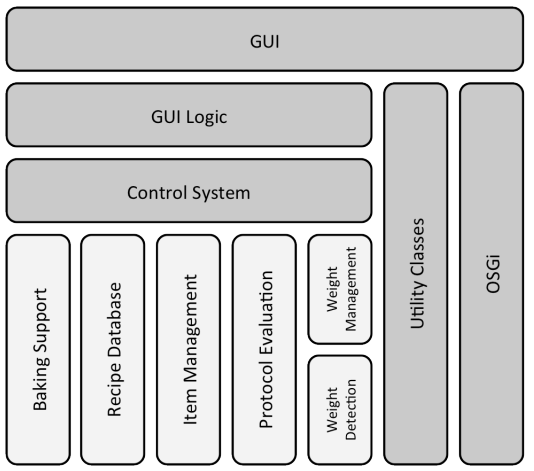

Figure 1: Smart Cooking Environment Architecture (SCEA)

A key question was how ingredients can be identified, weighed, and tracked. Identification can be done by various means, such as RFID tags or visual tags. RFID tags have several disadvantages. It is difficult to identify which items actually stand on the table, identify the location of this item on the table, and which item is held by a cook. Additionally, RFID tags are quite expensive which interferes with the costeffectiveness requirement. Therefore we choose visual tags that can be recognized by optical devices. As soon as an ingredient is placed on the table its tag will be recognized by the system. Each tag carries a unique ID which is used to identify corresponding product information.

The recipe retrieval service requires are mechanism that retrieves recipes from online repositories and matches recipe descriptions with available ingredients. Therefore fully semantically annotated recipe descriptions are required which are not available right now. A semi-formal tagging system is provided by microformats that knows the hrecipe schema (http://microformats.org/wiki/hrecipe). hrecipe encompasses attributes such as ingredients, instructions, and duration. Ingredient descriptions referenced by a hrecipe instance are used for comparison with available ingredients.

Requirements for the software architecture and abovementioned service requirements were used to identify OSGi as an appropriate basic architectural framework for SCEA. OSGi allows easy management of modules.

Based on OSGI, SCEA is based on the following set of modules that are used for realization of all services:

- Control System: controls interaction between all modules

- Recipe Database: XML-based repository of recipes retrieved from online repositories

- Recipe Processing: matches ingredients with recipes and tracks ingredients during formulation tasks

- Protocol Evaluation: processing of visual data used for ingredient identification and tracking

- Item Management: maintains the list of ingredients on the formulation table.

- GUI Logic: management of textual and visual presentation on the formulation table

- Weight Detection: interface between the internal scale and the weight management module

- Weight Management: maintains weights of ingredients on the formulation table

- Utility Classes: provides additional classes used by various modules, e.g., XML processing classes.

\section{IMPLEMENTATION}

The implementation of the SCEA architecture encompassed three areas: (1) hardware design and construction of the formulation table environment, (2) service implementation, and (3) integration.

The formulation table consists of a continuous glass plate $(72 \times 54 \mathrm{~cm})$. Identification and tracking of items on this table use an infrared installation integrated into the table. Good results are achieved when illumination is continuously distributed over the whole glass plate. For this, we use a multi-touch screen setup with a LEE color filter 225 for coating the plate and a LEE soft silver reflector 273 for coating the interior. For the infrared source an infrared LED emitter is used. Items are tagged with reacTIVision tags whose recognition is more robust than other 2D tags, such as QR tags. An infrared camera (Unibrain Fire-I COLOR BOARD Digital Camera) is used for tag recognition.

Information is projected onto the table by using a beamer (resolution 1024x768). A compact design was achieved by redirection via a mirror. 
A key element is the weighing mechanisms. At first, we tried to measure all four points of the glass plate but for cost and efficiency reasons we choose a design with just one scale. Based on invariants 1,2 and 3, the systems knows which items are on the table and which item has been lifted by the cook. Therefore it is sufficient for the weighing service to only use the relative increase of the overall weight. The inner part of the table with the glass plate rests on a scale (PCE-PS $75 \mathrm{XL}$ ) that provides a RS232 interface for USB.

All software services have been implemented as OSGi modules. For the recipe retrieval service, we use a recipe repository that annotates recipes by the hrecipe microformat schema (www.essen-und-trinken.de). If ingredients, e.g., a steak and a bag of potatoes, are placed on the formulation table, the system sends a URL to this web site with a list of recognized ingredients appended, e.g., http://www.essenund-trinken.de/suche/rezept/1?search type=profi\&

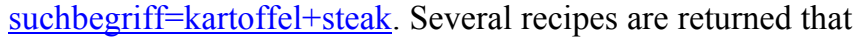
contain hrecipe information. After translation into XML, RDFa tags are identified and extracted. Each instruction has an ID that is used in description of instructions. By this mechanism all ingredient representations are grounded in physical ingredients with the help of reacTIVision tags. Missing ingredients are listed on the left side. Nonetheless the cook can immediately start with preparation and concocting tasks but the system will demand an ingredient if it is missing on the table.

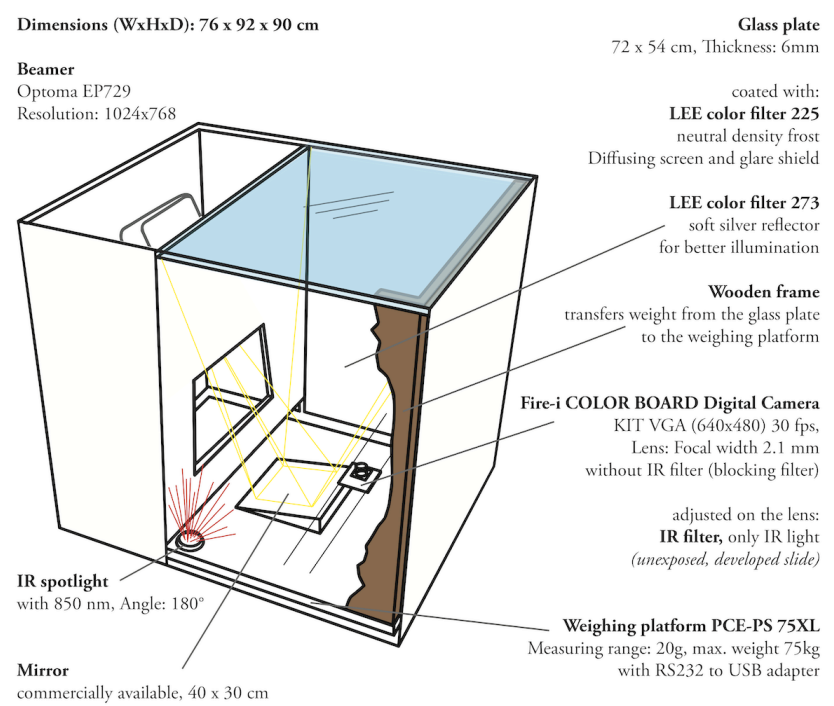

Figure 2: formulation table environment with scale mechanism

After a particular recipe has been selected it is shown on the left side of the table. The layout uses a simplified, easyto-use web site logic. Each step is highlighted so that the cook knows the current activity (recipe tracking service). All information presentations on the table are implemented by a Flash interface using the JFlashPlayer API (http://www.jpackages.com/jflashplayer).

The ingredient identification service visually encircles the particular ingredient. If an ingredient shall be used for the next activity it will be highlighted by a green circle. If the cook lifts a different ingredient a message will appear on the information pane on the left side that implements the alarm service.

The weighing service provides a bar that is filled in realtime till the appropriate amount has been added. Weighing is implemented with the scale and the RXTX Java library (http://rxtx.qbang.org).

Imagine a cook intends to bake a cake but he does not know exactly which one. He places a tagged package of flour, sugar, eggs, rum, and milk on the formulation table. The system sends a URL request (http://www.essen-undtrinken.de/suche/rezept/1?search type=profi\&suchbegriff $=\mathrm{V}$ anillin+rum) and receives a list of recipes. The cook selects a recipe, e.g., http://www.essen-und-trinken.de/rezept/146947/ feiner-marmorkuchen.html. This recipe information within the HTML page is transformed into a XML data structure (excerpt as follows):

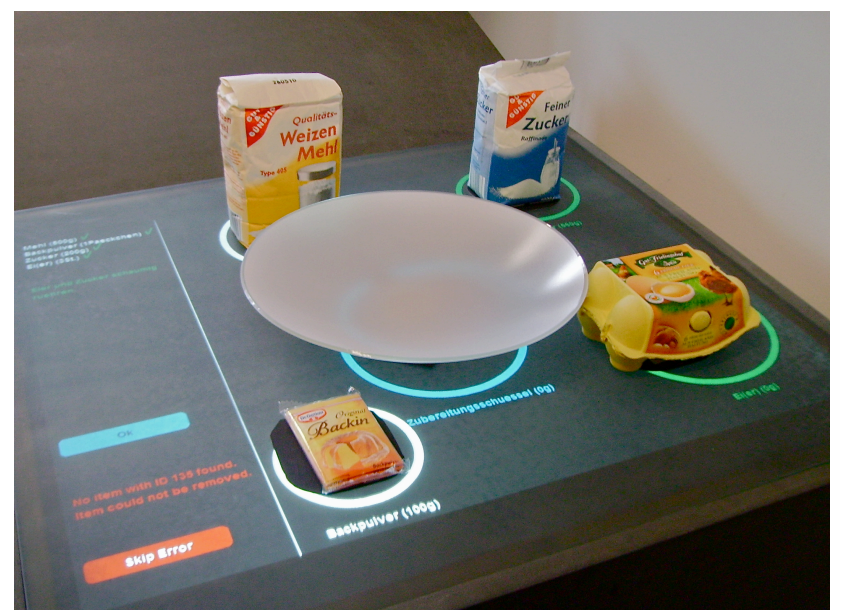

Figure 3: formulation table with two ingredients and visual markers

\section{EXAMPLE}

$<$ recipe $>$

$<$ title $>$ fine marble cake ${ }^{1}</$ title $>$

$<$ ingredients $>$

$<$ ingredient $\mathrm{id}=" 0 ">$

$<$ amount $>250<$ /amount $>$

$<$ type $>$ g $</$ type $>$

$<$ name $>$ butter or margarine $</$ name $>$

$[\ldots]$

$<$ steps $>$

$<$ step $>$

$<$ requires $>$

$<\mathrm{id}>0</ \mathrm{id}>$

1 Manually translated into English language. 
$<\mathrm{id}>1</ \mathrm{id}>$

$<\mathrm{id}>2</ \mathrm{id}>$

$</$ requires $>$

$<$ text $>$ Stir soft butter and add sugar, vanilla sugar, salt, eggs, and rum or brandy step by step. $</$ text $>$

$</$ step $>$

$[\ldots]$

$</$ recipe $>$

The RDFa-XML data structure of this recipe is loaded into the system. Other recipes might require additional $50 \mathrm{ml}$ of milk. The cook takes the milk bag and pours it into the boiler till the bar is filled. In the case of liquids, volumes are automatically translated into weights according to its specific weight.

\section{DISCUSSION}

The smart formulation table supports specific preparation and concocting tasks for cooking as described by Otl Aicher. The whole design has been carefully derived from domain knowledge and requirements. Formulation tasks were dissected into activities. On the level of activities, we made assumptions about which activities can be either improved or omitted by innovative services. Hence, our design distinguishes between a social system, an information sphere, a service system, and a realization system. The social system of cooking includes the cook(s), ingredients, tasks, and activities. The information sphere encompasses all cooking related semantics and information objects, such as recipes. Information objects are provided by services that are defined on service level. Services are designed to support tasks and activities of a social system. Social systems and service systems are realized by various technologies defined by the realization system. The formulation environment presented here is a good example how this four-folded systems framework can be used for step-wise design of intelligent environments.

On system level we found that adjusting the infrared installation with homogeneous illumination without reflections is a challenge. The dissection between infrared light for item recognition and tracking and optical light for information presentation provides a strong design principle for Tangible User Interfaces in general.

Whether invariants 1 and 3 are a threat for technology acceptance of our formulation table environment will be evaluated by empirical studies. Both invariants could be dropped if ingredients are additionally managed by RFIDtagged logistic systems. This would allow using ingredients without putting them on the formulation table first. But this poses a much higher complexity on the cooking environment. Therefore, these two additional invariants might be easier to accept than RFID-tagged environments.

\section{STATE OF THE ART}

Research within the realm of Ubiquitous computing used kitchen and cooking situations for exploring various kinds of services, such as personalization [6], advice giving [7], and technologies, such as barcodes [8], RFID tags [9-11] or planbased approaches [12].

As part of the Aware Home project, Tran et al. built a system called Cook's Collage that realizes the recipe tracking service by a video-based approach [1]. If a cook interrupts his/her cooking (s)he will be provided with a memory aid in form of a video summary of what (s)he has already done. In stress situations, this memory aid has proven helpful.

Nakauchi et al. investigated complex situations in which robots support humans in a kitchen [2]. Ingredients are recognized with the help of RFID tags. Advise is given by simple voice messages and gestures.

Terrenghi et al. developed a system that records complete stories of cooking meals. Emphasis has been laid on a qualitative design process [13].

Schneider describes SmartKitchen that uses various technologies within a kitchen [14]. Food containers are equipped with RFID tags. Weights are derived by an additional stationary scale. A Semantic Cookbook records cooking processes that can be shared with others.

Kranz et al. present two new devices for a context-aware kitchen: an augmented cutting board and a sensor-enriched knife [15]. Weight and acceleration data gained by the cutting board is used for identification of the type of food. Similarly when cutting food, the knife provides data that is characteristic for different types of food.

\section{CONCLUSION AND OPEN ISSUES}

Supporting everyday situations and activities is challenging and rewarding research objective for various research communities, such as Ubiquitous Computing, Pervasive Computing, and Semantic Technologies. We have presented an approach how to carefully derive technologically supported services for everyday situations within the domain of cooking with a special emphasis on preparation and concocting phases.

Currently we are conducting empirical studies based on technology acceptance models $[16,17]$ that investigate the intention to use the formulation table in relation to traditional formulation environments for cooking.

In this paper we have emphasized the design and service implementation side of the formulation table. In another project we have developed a middleware for semantically annotated product information based on the semantic web language OWL. With this, we can process recipes from different providers. Ribeiro et al. have developed an domain cooking ontology that needs to be populated by more specific concepts and relationships [18]. With a full-fledged cooking ontology we can translate between recipes of different providers, do reasoning on compounds of 
ingredients, and provide semantic representations for higherorder services, such as Natural Language Processing and

\section{REFERENCES}

[1] Q. T. Tran, et al., "Cook's Collage: Deja vu display for a home kitchen.," presented at the Proceedings of Home Oriented Informatics and Telematics (HOIT), 2005.

[2] Y. Nakauchi, et al., "Intelligent kitchen: cooking support by LCD and mobile robot with IC-labeled objects," in Intelligent Robots and Systems, 2005. (IROS 2005). 2005 IEEE/RSJ International Conference on, 2005, pp. 1911-1916.

[3] P.-y. Chi, et al., "Enabling nutrition-aware cooking in a smart kitchen," presented at the CHI '07 extended abstracts on Human factors in computing systems, San Jose, CA, USA, 2007.

[4] O. Aicher, Die Küche zum Kochen: Werkstatt einer neuen Lebenskultur, 2 ed. München: ökobuch Verlag, 2005.

[5] K. Fishkin, "A taxonomy for and analysis of tangible interfaces," Personal and Ubiquitous Computing, vol. 8, pp. 347-358, 2004.

[6] B. Li, et al., "Intelligent Oven in Smart Home Environment," in Research Challenges in Computer Science, 2009. ICRCCS '09. International Conference on, 2009, pp. 247-250.

[7] M. Rothensee, "A high-fidelity simulation of the smart fridge enabling product-based services," in Intelligent Environments, 2007. IE 07. 3rd IET International Conference on, 2007, pp. 529-532.

[8] L. Suhuai, et al., "Smart Fridges with Multimedia Capability for Better Nutrition and Health," in Ubiquitous Multimedia Computing, 2008. UMC '08. International Symposium on, 2008, pp. 39-44.

[9] R. Voyles, et al., "Smart tupperware: Active containers for kitchen automation," in SICE Annual Conference, 2008, 2008, pp. 3065-3069.

[10] M. Darianian and M. P. Michael, "Smart Home Mobile RFID-Based Internet-of-Things Systems and Services," in Advanced Computer Theory and Engineering, 2008. ICACTE '08. International Conference on, 2008, pp. 116-120.

[11] J. A. Ali, et al., "U-kitchen: application scenario," in Software Technologies for Future Embedded and Ubiquitous Systems, 2004. Proceedings. Second IEEE Workshop on, 2004, pp. 169-171.

[12] E. Kotsovinos and M. Vukovic, "su-chef: Adaptive coordination of intelligent home environments," in Autonomic and Autonomous Systems and International Conference on Networking and Services, 2005. ICAS-ICNS 2005. Joint International Conference on, 2005, pp. 74-74.
Robotics.

[13] L. Terrenghi, et al., "A cube to learn: a tangible user interface for the design of a learning appliance," Personal and Ubiquitous Computing, vol. 10, pp. 153-158, 2006.

[14] M. Schneider, "The semantic cookbook: sharing cooking experiences in the smart kitchen," in Intelligent Environments, 2007. IE 07. 3rd IET International Conference on, 2007, pp. 416-423.

[15] M. Kranz, et al., "Context-aware kitchen utilities," presented at the Proceedings of the 1st international conference on Tangible and embedded interaction, Baton Rouge, Louisiana, 2007.

[16] F. D. Davis, "Perceived Usefulness, Perceived Ease of Use, and User Acceptance of Information Technology," MIS Ouarterly, vol. 13, pp. 319-339, 1989.

[17] G. C. Moore and I. Benbasat, "Development of an instrument to measure the perceptions of adopting an information technology innovation," Information Systems Research, vol. 2, pp. 173-191, 1991.

[18] R. Ribeiro, et al., "Cooking an Ontology," ed, 2006, pp. 213-221. 